Revista de Investigación Educativa 27

julio-diciembre, 2018 | ISSN 1870-5308 | Xalapa, Veracruz

Instituto de Investigaciones en Educación | Universidad Veracruzana

\title{
Temáticas, geografías y debates en el campo de la pedagogía de la alternancia
}

\section{Topics, Geographies and Discussions in the field of Pedagogy of Alternation}

\author{
María Amalia Miano ${ }^{a}$ \\ Erik S. Lara Corro ${ }^{b}$
}

Recibido: 02 de enero de 2018

Aceptado: 18 de abril de 2018

En este artículo mapeamos la producción teórica elaborada en los últimos siete años sobre la pedagogía de la alternancia, con el fin de exponer un estado del conocimiento actual en este ámbito. Metodológicamente, el mapeo siguió dos ejes: (a) la agrupación semántica de las producciones de acuerdo con las temáticas recurrentes para dar cuenta de qué se ha estudiado y los tipos de producciones, y (b) el agrupamiento con un enfoque geográfico para reconstruir la cantidad de producciones y temáticas recurrentes por país. Nuestros resultados muestran que este campo de investigación aloja perspectivas muy diversas e incluso contrapuestas, entre las que pueden identificarse los polos "pedagogía de la alternancia y emancipación" y "pedagogía de la alternancia y sujeción”.

Palabras clave: Educación media; educación rural; campos conceptuales; semántica; geografía.

\footnotetext{
a Doctora en Ciencias Sociales. Investigadora Asistente, Centro de Estudios Desigualdades, Sujetos e Instituciones, Consejo Nacional de Investigaciones Científicas y Técnicas, Argentina. 
We map the theoretical production elaborated in the last seven years on the Pedagogy of Alternation, in order to expose a state of current knowledge in this field. The mapping followed two axes: (a) the semantic grouping of the productions according to the recurrent themes to account for what has been studied and the types of productions, and (b) the grouping with a geographic focus to reconstruct the quantity of productions and recurrent themes by country. The analysis shows that the field of pedagogy of alternation has diverse and opposing perspectives, in which highlight the poles "pedagogy of alternation and emancipation" and "pedagogy of alternation and subjection".

Keywords: Secondary school; rural education; conceptual frames; semantic; geography.

\title{
Temáticas, geografías y debates en el campo de la pedagogía de la alternancia
}

\author{
Topics, Geographies and Discussions in the \\ field of Pedagogy of Alternation
}

\section{Introducción}

$\mathrm{L}$ a implementación del modelo de agricultura industrial (Teubal, 2006), caracterizado principalmente por el cultivo de la soja transgénica y la concentración de la propiedad de la tierra y el mercado agroalimentario en pocas empresas transnacionales, ha generado un proceso de despoblamiento muy marcado de los contextos rurales. Esta cuestión ha sido documentada no solamente para los países de América Latina (Gras, 2017; Merenson \& Garaño, 2015; Nogar \& Jacinto, 2010), sino también para el resto del mundo (Altvater, 2012; Mooney, 2002). Entre algunos de los problemas que se plantean a la población rural en su conjunto, se ubica la preocupación por la permanencia de los jóvenes en estos ámbitos, cuestión que muchas veces entra en 
contradicción con las posibilidades que los mismos tienen de dar continuidad a sus estudios, ya que para lograr esa continuidad es necesario trasladarse muchas veces a grandes centros urbanos que cuenten con escuelas de nivel medio o secundario. ${ }^{1}$

La situación que atraviesa hoy la población rural constituye un problema no resuelto desde que la escuela pasó a ser el lugar de acreditación educativa, y aparece a lo largo de la historia y la geografía en muchos países, tal como sucedió, por ejemplo, en la década de 1930 en la localidad de Sérignac-Péboudou, una pequeña aldea del suroeste francés. Si bien se trataba de otro contexto histórico marcado principalmente por las posibilidades de reconstrucción económica y social luego de la Primera Guerra Mundial, en ese momento se visibilizaron las alternativas opuestas para los jóvenes campesinos de seguir trabajando en las actividades productivas realizadas por sus familias o bien irse del campo para poder seguir estudiando. Dinova (1997) ha documentado que, en esa ocasión, un grupo de familias pertenecientes al Sindicato Agrícola y a la Secretaría Central de Iniciativas Rurales (organización creada en 1920 ligada al catolicismo democrático) se acercó al párroco del pueblo con la preocupación de que sus hijos no querían estudiar porque preferían quedarse trabajando en las actividades que realizaban sus familias, situación también documentada por Duffaure (1994). Así, entre el párroco y las familias campesinas delinearon una modalidad de trabajo que implicaba la alternancia de la permanencia de los jóvenes una semana en la parroquia y otra en sus hogares.

A partir de esta iniciativa, comienza a implementarse una forma educativa que tiene un origen socio-comunitario y familiar. Si bien la lucha por los derechos educativos de los hijos por parte de las familias y las organizaciones comunitarias se encuentra como ejemplo constante en la literatura educativa (Baraldo, 2010; Gluz, 2013; Michi, 2010), en este caso, la novedad de esta modalidad escolar trajo dos cuestiones importantes. Por un lado, una estructura institucional que reconoce la participación de las familias en igualdad de derechos junto a los docentes (de allí que las primeras escuelas francesas se denominaran Maisons Familiales Rurales [MFR], es decir, las casas de las familias rurales) y, por otro, una pedagogía que se caracteriza por tener en cuenta el espacio y el tiempo como categorías de la enseñanza, en la cual tanto el predio escolar como el familiar y comunitario se consideran como espacios de aprendizaje. De esta manera, la alternancia, al mismo tiempo que facilita el arraigo de las

1. Por ejemplo, en Argentina la educación obligatoria comprende desde el nivel inicial hasta el fin del secundario o un total de 14 años de escolarización, pues a la obligatoriedad fijada por la Ley 26.026 se sumó la obligatoriedad de dos años de educación inicial por la Ley 27.045. 
familias, propone a los estudiantes interrogar su propia realidad y generar proyectos de vida que valoren la pertenencia a lo rural. En este proceso, el aprendizaje permite articular teoría y práctica, educación y trabajo, mientras que la realización de actividades productivas se convierte en un eje característico de esta pedagogía.

En 1950 ya existían en Francia 150 MFR distribuidas por todo el territorio de este país (Duffaure, 1994) y hacia principios de la década de 1960 comienzan a abrirse escuelas de alternancia en otros continentes, comenzando por África. En la actualidad, existen en el mundo aproximadamente mil trescientas escuelas de alternancia distribuidas en cuarenta países en cuatro continentes (América, África, Europa y Asia). ${ }^{2}$

El propósito de este artículo es mapear la producción teórica sobre la pedagogía de la alternancia, con el fin de dar cuenta de un estado del conocimiento actual en este campo de investigación. Hemos corroborado que no existe hasta el momento un trabajo de sistematización de este tipo. ${ }^{3}$ Así, partimos de las siguientes preguntas descriptivas con el fin de comprender algunas tensiones y debates que se dan en el marco de esta pedagogía: ¿qué trabajos se han escrito hasta el momento sobre la pedagogía de la alternancia?, ¿cuáles son las temáticas recurrentes en esos trabajos?, y ¿̇cuáles son los países que cuentan con producción teórica respecto a las escuelas de alternancia?

\section{Orientación metodológica}

El mapeo realizado en este artículo forma parte de una línea de investigación ${ }^{4}$ cuyo objetivo general consiste en describir propuestas educativas de nivel medio que se orientan al desarrollo local y la auto-gestión en ámbitos rurales en Argentina. Para esto, se han tomado dos ejes de indagación: por un lado, el enfoque educativo de estas

2. Dato aportado por la Asociación Internacional de los Movimientos Familiares de Formación Rural (AIMFR) en el Seminario Internacional "La metodología de la alternancia educativa como herramienta eficaz para la inserción laboral de los jóvenes del medio rural” ( 23 de junio de 2016, Buenos Aires, Argentina).

3. El único antecedente que hemos identificado es el trabajo de Teixeira, Bernartt y Trindade (2008), donde se sistematiza la producción teórica realizada en Brasil sobre la pedagogía de la alternancia, pero teniendo en cuenta únicamente tesis de doctorado y maestría realizadas en ese país.

4. En el marco del Proyecto de Investigación Científico Tecnológica 0943 "Comprender el aprendizaje colaborativo de carácter cooperativo-solidario. Sus pedagogías y dispositivos específicos”, dirigido por la Dra. Ana Inés Heras y financiado por la Agencia Nacional de Promoción Científica y Tecnológica. 
escuelas (ligado a la implementación de herramientas e instrumentos pedagógicos distintivos) y, por otro, la organización y gestión institucional (ligada a la figura de la co-gestión entre comunidad y Estado).

Hemos iniciado un trabajo de campo en el mes de octubre de 2016 en una escuela de alternancia de la provincia de Buenos Aires. Durante 2017, asistimos a las reuniones quincenales del Consejo de Administración de esta escuela, con el fin de documentar las prácticas que caracterizan a la co-gestión. También, llevamos a cabo una estancia breve en la primera escuela de alternancia de la Argentina, situada al norte de la provincia de Santa Fe.

A través del desarrollo de estas actividades, realizamos hasta el momento un total de catorce entrevistas a diferentes actores sociales vinculados a las escuelas de alternancia en las provincias de Buenos Aires y Santa Fe (docentes, estudiantes, directivos de las escuelas, padres y madres de estudiantes, miembros de las organizaciones de segundo grado que nuclean a las escuelas y también personas que han participado de la apertura de estas escuelas en la Argentina). También contamos con un archivo fotográfico que registra la vida cotidiana de la primera escuela de alternancia de Argentina desde 1970 hasta la actualidad. Finalmente, generamos un cuaderno de campo con las observaciones de las reuniones del Consejo.

Al indagar sobre los orígenes de las escuelas de alternancia en nuestro país, hemos observado que los vínculos con experiencias de escuelas de alternancia abiertas en otros países han sido un factor decisivo para las posibilidades de apertura de escuelas de este tipo en Argentina, tal como lo hemos relevado en Heras y Miano (2017). Esta mirada hacia otras geografías y momentos nos llevó a la necesidad de contextualizar nuestras preguntas y objetivos de investigación en el campo de la producción teórica de la alternancia. De esta manera, hemos constatado que no existen hasta el momento trabajos de revisión como el realizado aquí, de ahí la importancia del mapeo elaborado en este texto.

Entendemos aquí al mapeo como un organizador que es al mismo tiempo epistémico (Sagastizábal, Burin, Heras \& Miano, 2006) —en tanto posibilita generar nuevos conocimientos a partir de visualizar, agrupar y organizar la produccióny semántico. Asimismo, nos permite establecer relaciones (de inclusión, exclusión, complementariedad) de acuerdo con el contenido desarrollado en cada uno de los trabajos. En tanto mapa, nos permitirá situarnos en el espacio conceptual de la pedagogía de la alternancia y desplegar sus líneas, las articulaciones entre ellas y algunos debates aún implícitos en este campo. 
El mapeo que realizaremos en este trabajo seguirá dos ejes. Primero, una agrupación semántica de las producciones de acuerdo con las temáticas recurrentes que surgen de los mismos, para dar cuenta de qué se ha estudiado y qué tipos de producciones son (libros, artículos en revistas, ponencias). Esta agrupación semántica nos ha llevado a identificar algunas categorías para agrupar a los trabajos que se relacionan entre sí por estar cruzados por determinado eje temático predominante. Luego, hemos realizado un trabajo de agrupamiento con un enfoque geográfico para poder reconstruir la cantidad de producción y temáticas recurrentes por país, cruzándolo a su vez con la cantidad de años que la alternancia está presente en los mismos. Este tipo de procedimiento permitirá visualizar y superponer analíticamente distintas capas del campo de la pedagogía de la alternancia: la espacial o geográfica, la temporal y la referida a la producción conceptual en este campo.

Hemos realizado búsquedas en distintas bases de datos, repositorios y editoras (ScienceDirect, JSTOR, Springer, SAGE Journals), así como en diversas revistas (Revue Française de Pédagogie; Ela, Études de Linguistique Appliquée, Educação e Pesquisa, Revista de Ciências Humanas, Revista Extensão Rural, entre otras). Esta búsqueda especializada nos ha remitido a comunicaciones de diverso tipo. En su mayoría, se trata de artículos de revistas científicas, pero también y en menor medida, encontramos tesis de maestría y doctorado y ponencias presentadas en Jornadas y Congresos.

La búsqueda de los trabajos se realizó a través de distintos descriptores. El primero de ellos, más general y abarcador que los siguientes, se enfocó en los términos: "escuelas de alternancia” y "pedagogía de la alternancia”. Esta indagación nos arrojó un total de 33 artículos de revistas especializadas, 19 ponencias, seis tesis de posgrado y una tesina de grado, dos capítulos de libro, siete libros y un informe de trabajo.

La segunda búsqueda estuvo acotada a los nombres que adoptan las escuelas de alternancia en cada uno de los países que cuentan con este tipo de escuelas. Cabe aclarar que en cada país los nombres de las escuelas varían e incluso hay países que cuentan con más de un sistema de escuelas de alternancia (por ejemplo, en Brasil se encuentran las Escolas Família Agrícola y las Casas Familiares Rurais; en Argentina están los Centros Educativos para la Producción Total, las Escuelas de la Familia Agrícola y los Centros de Formación Rural). Esta exploración a través de las denominaciones de las escuelas en cada país nos arrojó un total de 37 textos, de los cuales 18 son artículos de revistas, 11 son ponencias, dos son capítulos de libros, dos libros, un informe, dos tesis de postgrado y una tesina de grado. De estos textos, 23 son en idioma portugués, once en español, dos en francés y uno en italiano. 
Por último, la tercera búsqueda se realizó tomando como referencia el cruce de algunos descriptores tales como: participación, familias, actores sociales, perspectiva multi-actoral, actor-red, cruzados con los nombres de las escuelas por país. Esta búsqueda estuvo focalizada en el aspecto ligado a la co-gestión entre familias-comunidad y Estado en las escuelas de alternancia. Esta averiguación arrojó un total de 47 artículos, de los cuales 20 son ponencias, 22 son artículos de revistas, dos son capítulos de libros, dos libros y una tesis de maestría.

Posteriormente leímos todos los artículos (102 en total, quitando algunos trabajos repetidos en las búsquedas a través de los diversos descriptores) y agrupamos semánticamente la producción bajo cinco categorías distintas. Como indicamos más arriba, en este caso la relación semántica es la de la inclusión de los textos bajo determinada categoría a partir de la similitud en cuanto al tema abordado en cada texto. La inclusión bajo determinada categoría excluye al texto de poder ser incorporado en otra, más allá de que luego en las conclusiones realizaremos una articulación de las diversas categorías. Para la creación de las categorías hemos seguido el procedimiento de la teoría enraizada en datos (Glaser \& Strauss, 1967), que implica un nivel de generalización a partir de la lectura de la información. Esta agrupación semántica nos permitirá realizar una organización temática de la producción y es lo que desarrollaremos en el próximo apartado. En el apartado cuatro hemos reagrupado esos textos desde un eje geográfico, para reflejar en términos cualitativos y cuantitativos la distribución de la producción del campo de la alternancia por país.

En los resultados nos focalizaremos en los artículos desarrollados entre 2011 y 2017 con el fin de que el mapeo dé cuenta de un estado del arte actual en el campo de la alternancia. De esta manera, de los 102 artículos relevados, nos centraremos en los 59 que entran en el corte temporal mencionado.

\section{Agrupación semántica de los textos}

A partir de la lectura y análisis del contenido de los 59 artículos que entran en el corte temporal mencionado, podemos agrupar semánticamente a los mismos en función de estas cinco categorías construidas a partir de esa lectura: 1) Pedagogía de la alternancia y desarrollo local-rural (con un total de 14 textos); 2) Desarrollo del enfoque pedagógico de la alternancia, centrándose en sus instrumentos específicos (14 textos); 3) La pedagogía de la alternancia y la conformación de un sujeto crítico y arraigado al 
medio rural (12 textos); 4) Análisis histórico de procesos de apertura de escuelas de alternancia en distintos países y regiones (9 textos), y 5) Pedagogía de la alternancia y educación para el trabajo (8 textos). A su vez, hemos hallado dos textos que no podemos incluir en ninguna de las categorías anteriores. ${ }^{5}$

Cabe aclarar que en algunos trabajos las categorías 1 y 3 se encuentran relacionadas, ya que se supone que si la pedagogía de la alternancia contribuye a conformar un sujeto crítico respecto al modelo de desarrollo imperante en el mundo rural (lo cual se ve como contrario al tipo de producción característico de la agricultura familiar), esto a su vez contribuye al desarrollo local y rural. Sin embargo, preferimos mantener dos categorías diferenciadas ya que consideramos que los textos que se incluyen en la categoría 3, explícitamente manifiestan un componente de tipo político, ligado a las posibilidades que tiene la pedagogía de la alternancia de fortalecer la lucha y resistencia de los campesinos frente al desarraigo ocasionado por nuevas formas de uso y explotación de la tierra en los espacios rurales (Feito, 2014), cuestión que no queda del todo explicitada en los textos que incluimos bajo la categoría 1.

Como hemos sostenido, el mapeo sirve para visualizar, agrupar y organizar, a la vez que permitirá desplegar algunas articulaciones y tensiones, tanto hacia dentro de cada categoría como con las demás. A continuación, desarrollaremos los aspectos más destacados de cada una de las categorías relevadas, resaltando los enfoques principales adoptados por los trabajos agrupados en cada una de ellas.

\section{Desarrollo y especificación de cada categoría emergente}

\subsection{Pedagogía de la alternancia y desarrollo local-rural}

En esta categoría se encuentran aquellos trabajos que dan cuenta de las maneras en que la pedagogía de la alternancia permite contrarrestar los desplazamientos de las poblaciones rurales hacia las grandes ciudades como consecuencia de la producción

5. Nos referimos al texto de Costa y Doula (2014) que se ubica en el contexto de los estudios sobre juventud y busca responder a la pregunta “¿qué es ser joven para los estudiantes de la EfA Paulo Freire?” Las autoras comentan que el interés por conocer a los jóvenes de los ámbitos rurales es algo reciente, a diferencia de lo que ocurre con los jóvenes de ámbitos urbanos; también al texto de Bernartt y Pezarico (2011) donde analizan un programa de incentivo realizado en Brasil para la formación científica de estudiantes de Mozambique, Cabo Verde y Angola (todos países africanos de habla portuguesa). El programa consistió en una estadía de estudiantes africanos en la Universidad Tecnológica Federal de Paraná, formándose en temáticas vinculadas con la pedagogía de la alternancia. 
a gran escala, la concentración de la propiedad de la tierra, la mecanización y la aplicación de agro-tóxicos. Si bien todos los textos trabajan sobre este supuesto, podemos encontrar algunos enfoques diferenciales que desarrollaremos a continuación. En primer lugar, dentro de esta categoría podemos agrupar un conjunto de textos que mencionan que la pedagogía de la alternancia permite convertir a lo escolar en un instrumento para el desarrollo (Aldana, 2012; Pereira, 2016; Rotman, 2015).

En segundo lugar, encontramos textos que enfocan al desarrollo local desde el accionar y la perspectiva de los estudiantes y egresados de las escuelas de alternancia. De esta manera, se trabaja sobre las expectativas a futuro de los estudiantes (Lins \& Cavalcante, 2011); sobre las posibilidades de permanencia de los jóvenes en el campo (ya sea durante el cursado de la escuela secundaria como así también una vez que egresan de ella) (Lamas, 2016; Piovezana \& Oliveira, 2012; Queiroz Fraga \& de Sousa, 2011; Texeira, 2013)y sobre la forma en que los conocimientos de los jóvenes egresados repercuten en la comunidad local promoviendo el desarrollo (do Carmo \& dos Prazeres, 2011). La mayoría de estos trabajos toma como criterio de logro de desarrollo local a la cantidad de egresados de estas escuelas que permanecen en el medio rural una vez finalizados sus estudios secundarios.

En tercer lugar, algunos textos enfocan la manera en que la alternancia promueve el desarrollo local a través de la conformación de redes sociales en la comunidad. Así, el acento está puesto en dar cuenta de los vínculos interinstitucionales que crean las escuelas con organismos locales y regionales (Lorenzo, 2012; Novais, 2014).

Finalmente, encontramos textos que se refieren en específico a cuestiones de tipo medio ambientales involucradas en los procesos de desarrollo, argumentando que la pedagogía de la alternancia promueve en sus estudiantes prácticas ligadas al cuidado del ambiente. El tratamiento de las cuestiones ambientales se realiza de diferentes formas, por ejemplo, mediante el análisis de los proyectos productivos presentados por los estudiantes de una escuela de alternancia (Schneider, 2013); a través de la incorporación del concepto de sustentabilidad dentro del currículum y práctica pedagógica de estas escuelas (Conceição \& da Costa, 2012), y al analizar la convergencia entre agroecología y pedagogía de la alternancia, en tanto la primera implica poner en juego saberes tradicionales de los campesinos, los cuales, a través de instrumentos pedagógicos específicos de la alternancia, se conforman en un cuerpo de saberes centrales dentro del currículum de esta pedagogía. Estos saberes tradicionales, a partir de los cuales se apoya la enseñanza de la alternancia, serían contrapuestos a los desarrollos científicos ligados a la explotación agropecuaria a gran escala (Araújo, Linhares \& Teixeira, 2011). 
En síntesis, todos estos trabajos tienen en común el hecho de destacar la forma en que la pedagogía de la alternancia contribuye al desarrollo de los ámbitos rurales, a través de lograr el arraigo de los jóvenes en estos espacios, de la creación y fortalecimiento de redes sociales en las comunidades rurales y de las posibilidades de realizar proyectos productivos que cuiden el medio ambiente. Subyace a esta idea, también, el hecho de que esos jóvenes poseen además un tipo de formación específica que permitiría contrarrestar los efectos generados por el modelo de desarrollo hegemónico en el ámbito rural, que tiene como resultado la expulsión de los pequeños propietarios rurales, peones y campesinos.

\subsection{Desarrollo de los instrumentos pedagógicos de la alternancia}

Esta categoría se corresponde con aquellos trabajos que se centran en los lineamientos pedagógicos y didácticos distintivos de la alternancia. Como hemos mencionado, en las escuelas de alternancia se implementan instrumentos pedagógicos específicos para lograr articular práctica y teoría, formación y trabajo, espacio escolar y espacio familiar-comunal.

Encontramos algunos textos que destacan el potencial formativo y político del enfoque pedagógico de la alternancia, que se centra en partir de la interrogación de las propias condiciones de vida de los estudiantes (Bock \& Fagundes, 2014; Cardoso \& Hoff, 2013; Colatto, 2013). Desde esta perspectiva, la pedagogía de la alternancia es conceptualizada como una "pedagogía de resistencia cultural" (Silva, 2013, p. 119).

Metodológicamente, varios de los textos agrupados en esta categoría toman un instrumento pedagógico específico y lo analizan en profundidad, destacando muchas veces tanto las ventajas de la implementación de ese instrumento en el proceso de enseñanza y aprendizaje como también señalando algunas dificultades. Así, encontramos por ejemplo el trabajo de Kunrath (2012) que se centra en la formación por área de conocimiento presente en las escuelas de alternancia. Si bien esta orientación pedagógica es fructífera para articular saberes, la autora menciona que muchas veces los docentes tienden a enseñar más aquella disciplina para la cual están formados, descuidando las otras áreas. Bencke y Costa (2016) analizan el "estagio de vivencia", que consiste en un intercambio de estancia en las casas de familias entre alumnos de distintas escuelas de alternancia dentro de una misma región de Brasil. Para las autoras, este instrumento pedagógico permite el intercambio de saberes y hábitos fami- 
liares, pero genera algunos conflictos en cuanto a las diferencias de género (ligadas a que la mayor parte de las familias prefiere recibir estudiantes varones en lugar de mujeres). Un último ejemplo de trabajos que analizan en profundidad un instrumento pedagógico específico es el de da Silva, Andrade y Moreira (2015), quienes se centran en el "cuaderno de realidad", tomándolo como un género no usual en los espacios de aprendizaje. Se trata de un cuaderno que llevan los propios estudiantes, donde deben ir escribiendo sus aprendizajes, lo cual "les permite sistematizar y organizar los conocimientos escolares a través de textos” (p.361). Así, los autores se centran en los procesos de retextualización que deben producir los estudiantes al transformar un mismo texto desde y hacia diferentes soportes (por ejemplo, del habla a lo escrito, en el caso de estar haciendo una entrevista oral y de lo escrito al habla, en el caso en que deben explicar a otros o poner en común lo que escribieron). Los autores concluyen que, a través del cuaderno de realidad, las prácticas de escritura y de retextualización tienen un lugar importante en la pedagogía de la alternancia.

Otros trabajos agrupados en esta categoría evalúan determinados instrumentos pedagógicos tomando como eje transversal las posibilidades de articulación entre lo que se identifica como "conocimiento científico" y "conocimiento tradicional" (Lima \& Freixo, 2012), que es uno de los pilares de la pedagogía de la alternancia. Así, por ejemplo, Lima y Freixo argumentan que el "plan de búsqueda”, en tanto es una indagación que comienza en el espacio más cercano del estudiante (su familia, sus vecinos, su comunidad) pero que luego se va reelaborando al pasar por diversas instancias (búsqueda bibliográfica, consulta a expertos en las temáticas abordadas), es un instrumento que promueve el diálogo entre distintos tipos de saberes. En la misma línea, Assunção y Borges (2012) evalúan en qué medida los saberes que se enseñan sobre las matemáticas a los estudiantes de una escuela de alternancia tienen que ver con el propio contexto rural, y concluyen que esos saberes están vinculados con las actividades productivas que realizan las familias, en este caso, ligadas a la pesca y la agricultura. También, Piatti (2014) analiza la forma en que el cuaderno de realidad se convierte en un soporte en el cual los estudiantes comienzan a articular los saberes científicos con los de la vida cotidiana.

Finalmente, encontramos textos que destacan algunas aristas específicas de los instrumentos pedagógicos como, por ejemplo, la utilización de las nuevas tecnologías de la información y la comunicación para mantener el contacto entre docentes y estudiantes, cuando estos últimos se encuentran en el periodo de permanencia en sus hogares (Lobo, 2011); la forma en que algunos egresados aplican ciertos instrumen- 
tos pedagógicos en su vida laboral, por ejemplo, las dinámicas del trabajo en equipo (Teixeira \& Antunes, 2011), o bien la realización de una crítica global a la aplicación de algunos instrumentos pedagógicos que se analizan como desactualizados respecto a la velocidad de los cambios que ocurren en el contexto rural (Gómez, 2011).

Como podemos ver, esta categoría agrupa textos y enfoques muy diversos. Desde la valorización de los instrumentos pedagógicos de la alternancia, en tanto permiten mejorar la calidad de vida de los estudiantes y sus familias al partir de interrogar y trabajar sobre su propia realidad, hasta la crítica de estos instrumentos por encontrase desactualizados frente a los cambios vertiginosos experimentados en los ámbitos rurales.

\subsection{La pedagogía de la alternancia y la conformación de un sujeto crítico y arraigado al medio rural}

Dentro de esta categoría ubicamos aquellos trabajos que consideran que la alternancia ofrece a los campesinos herramientas para resistir al proceso de expansión del capitalismo en el campo, el cual excluye a los trabajadores rurales de sus derechos sociales y los expulsa de sus tierras. En un trabajo anterior (Heras \& Miano, 2017), se ha dado cuenta de este proceso caracterizándolo como una doble dinámica de la acumulación (de bienes, tierra, capital, titulaciones) por desplazamiento y desposesión. A su vez, se sostuvo que estos procesos no están exentos de resistencias generadas por grupos que defienden sus ideas, territorios, formas de vida y producción. Las escuelas de alternancia se constituirían en un espacio de conformación de un sujeto crítico, arraigado a su medio rural y que desarrolla esos procesos de resistencia a la acumulación desmedida. Es así que algunos autores asocian a la pedagogía de la alternancia con el concepto de praxis, por las posibilidades de transformación del medio rural (do Amaral, 2013; Santana da Silva, 2012).

Varios de estos trabajos presentan una diferenciación entre la educación rural, pensada desde lo urbano, cuyo objetivo es la domesticación y reproducción del orden social establecido, y la educación de campo (Educação do Campo), como una educación humana y emancipatoria, vinculada a la vida, al trabajo y la cultura de los campesinos (Almeida \& Germani, 2013; Chaves \& Foschiera, 2014; da Silva, 2015). En Brasil, las Escolas de la Familia Agrícola (EFA), las Casas Familiares Rurales (CFR) y las escuelas itinerantes del Movimento dos Trabalhadores Rurais Sem Terra MsT se enmarcan dentro de este movimiento de educación de campo. 
Uno de los principales ejes de estos trabajos es la lucha de los campesinos por permanecer en la tierra (de Lima, 2011), mencionando tanto la defensa del territorio inmaterial (cultura local) como el sostenimiento del territorio material, que es donde se da la reproducción de la vida (Almeida \& Germani, 2013). El correlato de esta lucha es el arraigo que es enfocado, por un lado, desde una perspectiva geográfica al considerar que las escuelas de alternancia sirven para reafirmar los vínculos que ligan a las personas con los lugares (Batista, 2015); por otro, desde una perspectiva pedagógica al analizar de qué manera algunos instrumentos, como el plan de búsqueda, integran la vida con la escuela y desencadenan la comprensión del significado político y social de los contenidos curriculares (Santana da Silva, 2012; Zanuncio \& Zanuncio, 2015).

También encontramos trabajos que destacan que el potencial político de la alternancia reside en la formación de estudiantes como agentes de cambio en sus contextos rurales (Almeida \& Batista, 2011; Santos \& Cardel, 2011). En tanto a través de su paso por la escuela se encuentran con varias instancias en las cuales tienen que exponer sus ideas frente a otros, los estudiantes se sienten más preparados para hablar en público, tomar decisiones, movilizar políticamente a la población local y luchar por mejores condiciones de vida y trabajo (Santos \& Cardel, 2011). Otro aspecto que matizan algunos textos es sobre cómo la formación crítica de los estudiantes tiene que ver con fortalecer una raíz cultural propia o valorizar un modo de vida específico (Novais, 2012; Sobreira \& da Silva, 2014), lo cual llevaría a que el campo sea visto no únicamente como un espacio de producción, sino como un espacio de vida, luchas, resistencia y manifestaciones culturales.

En síntesis, bajo esta categoría encontramos trabajos que toman diversos aspectos de la alternancia (su enfoque, sus instrumentos pedagógicos, las posibilidades que brinda para el arraigo) para conectarlos con su potencial político y transformador. De esta manera, los aspectos revisados en las anteriores categorías encontradas en el campo de la alternancia son valorados en función de sus posibilidades de fortalecer la lucha campesina.

\subsection{Análisis histórico de procesos de apertura de escuelas de alternancia en distintos países y regiones}

Bajo esta categoría agrupamos aquellos trabajos que realizan una reconstrucción histórica de la forma en que se han abierto escuelas de alternancia, ya sea en distintos países como en diversas regiones dentro de un mismo país. Metodológicamente, la 
mayor parte de estos trabajos se estructura alrededor de un caso específico, tomando como casuística ya sea la apertura de una escuela en particular o la implementación de la alternancia en una región dentro de un país (Oliva, 2016; de Oliveira, 2013; Pereira, 2015; Reis \& Etges, 2016; Scopel, Passos, Palaoro, Dos Santos \& Bernartt, 2011; Texeira, 2013).

Podemos observar dos grandes focos de interés dentro de los trabajos que realizan reconstrucciones históricas de casos de apertura de escuelas en países y regiones. El primero de ellos es el que se centra en dar cuenta de los diversos actores sociales que intervinieron en los momentos iniciales de apertura de estas escuelas. Es ejemplo de dicho enfoque el texto de Valadão, Neto y de Andrade (2017) adotaram-se a teoria do ator-rede (TAR que desarrolla las tensiones, acuerdos y posicionamientos establecidos entre diversos actores sociales (Estado, empresas, Consejo de Educación, movimientos sociales, universidad, Iglesia católica) para la expansión de la pedagogía de alternancia en la región de Rondonia (Brasil). También el de Fernández y Welti (2006), que realiza un recorrido similar al anterior pero para analizar el surgimiento de las escuelas de alternancia en la Argentina, destacando el rol del Movimiento Rural Católico, las familias campesinas y las Ligas Agrarias. ${ }^{6}$ El hecho de que la reconstrucción histórica ponga el foco en los diversos actores sociales que se articulan para dar lugar al surgimiento de la alternancia, da cuenta de otra de las características específicas de estas escuelas, que es su matriz comunitaria. Es decir, la alternancia surge a partir de las demandas concretas de un grupo de agricultores y se sostiene luego por esas articulaciones realizadas entre las escuelas y un conjunto amplio de sujetos y organizaciones sociales (Pacheco \& Simonini, 2016).

El otro foco de interés reside en aquellos trabajos que toman el caso de una escuela de alternancia en particular, para dar cuenta de algunas contradicciones que se plantean entre las prácticas que se dan en la escuela y las concepciones más generales de la pedagogía de la alternancia que operan como directrices. Así, por ejemplo, de Melo, Soares y Lucini (2013) muestran las dificultades que enfrentan las escuelas de alternancia en cuanto a la falta de financiamiento público, al estar institucionalmente configuradas a través del sostenimiento de las familias de los estudiantes. Otra con-

6. Se trata de organizaciones sociales y políticas que surgieron en Argentina a fines de 1960, que representaban las demandas de los campesinos frente a las políticas de corte neoliberal que comienzan a instalarse en el sector rural. Principalmente surgieron en las provincias del noreste argentino. Durante la última dictadura militar fueron desarticuladas y sus principales dirigentes sufrieron el encarcelamiento, la desaparición y el exilio (Calvo \& Percíncula, 2012). 
tradicción tiene que ver con el hecho de que las escuelas de alternancia forman para el arraigo en un contexto donde no se tiene la seguridad de la propiedad de la tierra y se percibe una alta mecanización de las actividades agrícolas. Siguiendo esta línea, por ejemplo, Jahn y Presotto (2013) analizan el caso de una Casa Familiar Rural abierta en Perola Oeste (Brasil) y constatan que sólo $30 \%$ de los egresados se queda a vivir en el campo.

Como podemos observar, varios trabajos dentro del campo teórico de la pedagogía de la alternancia adoptan un enfoque histórico que permite reconstruir las diversas etapas de apertura de una escuela o bien de varias escuelas en una misma región. Consideramos que poner el foco de interés en estos procesos es algo específico de esta pedagogía, pues a diferencia de las escuelas estatales, la apertura de escuelas de alternancia apela al accionar de una gran diversidad de actores sociales, tal como puede constatarse en la lectura de los textos agrupados bajo esta categoría. Las dinámicas de articulación de actores en cada caso específico son muy diversas, y aportan una gran riqueza para analizar las potencialidades y tensiones de los procesos conformados por múltiples actores como respuesta a las demandas de escolarización en contextos en los que el acceso a la educación se encuentra vulnerado.

\subsection{Pedagogía de la alternancia y educación para el trabajo}

Uno de los lineamientos pedagógicos centrales de la alternancia es el aprendizaje promedio de la práctica situada. Esto implica la realización de proyectos productivos ligados a las actividades que realizan las familias de los estudiantes. En relación con esto, los trabajos agrupados bajo esta categoría se enfocan en la alternancia como medio para la profesionalización de los estudiantes y su posterior inserción en el mundo del trabajo. En este sentido, la mayoría de los textos hace referencia a la inserción laboral de los egresados (véase, por ejemplo, Barbosa, 2014; Monge, 2016; Santos \& de Sousa, 2015).

Existe otro conjunto de trabajos que se enfocan en la didáctica de la alternancia para la formación profesional (Boudjaoui, Clénet \& Kaddouri, 2015; Frossard, 2014; Pentecoteau, 2012). Tanto en los textos de Boudjaoui et al. (2015) como en el de Pentecouteau (2012), el foco está puesto en la formación de competencias para el trabajo a través de la alternancia (muchas veces aplicada en el nivel de la formación universita- 
ria) y no se hace siquiera mención a las escuelas de alternancia. ${ }^{7}$ En tanto hay saberes que no pueden transmitirse a través de las palabras, el aprendizaje a través de situaciones concretas de trabajo, tal como se realiza en la alternancia, es postulado como un método adecuado para aprender competencias profesionales. El trabajo de Frossard (2014) analiza cómo la alternancia contribuye a la formación de técnicos agropecuarios (egresados de dos escuelas de alternancia, una en Argentina y otra en Brasil) capaces de intervenir en su realidad local. Finalmente, el trabajo de Santos (2015) hace referencia a algunas limitaciones con las que cuentan los egresados de una escuela de alternancia para su actuación profesional.

En esta línea de trabajos centrados en la didáctica de la alternancia para la formación profesional es que se han desarrollado las diversas tipologías en función del nivel de articulación entre lo educativo y el trabajo. De esta manera, Boudjaoui et al. (2015), retomando los desarrollos de otros autores, mencionan a la alternancia sin integración entre trabajo y formación, la cual implica dos actividades de aprendizaje, una de estudio y otra de trabajo sin relación entre sí; la alternancia con poca integración entre trabajo y formación, cuando el ámbito de trabajo es visto como un lugar de aplicación de los saberes teóricos o metodológicos, y la alternancia con fuerte integración entre trabajo y formación, que es cuando el medio laboral y el educativo plantean de forma conjunta situaciones, problemas y recursos. En el texto de Pentecouteau (2012) aparecen las mismas distinciones, aunque con otros nombres (alternancia yuxtapositiva, asociativa e integrativa).

Para finalizar, destacamos dentro de esta categoría el trabajo de Padawer, Greco y Rodríguez (2013) que se centra en analizar la transmisión inter generacional de saberes para construir "sucesores", en un contexto en el cual las generaciones jóvenes problematizan cotidianamente sus oportunidades de futuro ligadas a lo rural. Para las autoras, la conformación de sucesores "remite al aprendizaje que se produce cuando las jóvenes generaciones adquieren conocimiento acerca del ambiente en sus experiencias cotidianas de trabajo agrícola” (p. 51). Las escuelas de alternancia se conforman como un espacio que permite, desde su propuesta pedagógica, articular las experiencias formativas ligadas al entorno de los estudiantes.

En síntesis, los trabajos agrupados bajo esta categoría se centran en analizar las articulaciones entre la educación y el trabajo. Así encontramos aquellos textos que

7. En este sentido, hemos relevado que existen una gran cantidad de textos que se refieren a la articulación entre formación para el trabajo y alternancia sin hacer referencia explícita a las escuelas de alternancia. No profundizamos en ello en tanto no es el fin de este trabajo. 
enfocan la inserción laboral de los egresados de las escuelas en el trabajo del medio rural (sopesando incluso las transformaciones acaecidas en el mismo); los textos que analizan la forma en que la alternancia permite dar una continuidad a los saberes de las familias que trabajan en el medio rural, y los textos que extraen de la alternancia su didáctica de formar a través del hacer para la generación de competencias para el mundo laboral.

\section{Análisis de la producción bibliográfica de la alternancia desde un enfoque geográfico}

La búsqueda realizada en las diversas bases de datos mencionadas en el apartado metodológico nos permitió constatar que de los cuarenta países en los cuales hay actualmente escuelas de alternancia, sólo en ocho de ellos hemos hallado textos que reflexionen sobre esta pedagogía (Argentina, Brasil, Colombia, España, Francia, Guatemala, Italia y Perú). Ahora bien, al realizar el corte temporal de los textos producidos desde 2011, sólo encontramos cinco países en los cuales existe producción teórica, tal como puede verse en la Tabla 1.

Tal como se observa en la Tabla 1, Brasil encabeza cuantitativamente la producción en el campo de la pedagogía de la alternancia, seguido muy por detrás por Argentina. Luego, siguen Francia y Perú con dos textos cada uno, y finalmente Colombia, con un texto. Además, se puede observar que, excepto Francia, todos los países en los que actualmente se encuentran textos sobre la pedagogía de la alternancia son latinoamericanos. Si cruzamos estos datos con un eje cronológico, podemos observar que desde 2011 Brasil concentra la mayor cantidad de producción de bibliografía por año en comparación con los demás países, destacándose en esta materia los años 2011 y 2014.

En cuanto a Argentina, cuenta con cierta continuidad temporal en cuanto a la producción de trabajos, aunque en términos relativos con Brasil, la cantidad de textos por año es muy escueta. Es significativa la diferencia de producción entre Argentina y Brasil en el corte temporal que realizamos (2011-2017), notándose una expansión de los estudios de alternancia en Brasil en oposición a lo que ocurre en Argentina que, excepto en 2015 cuando se registran cuatro producciones, no supera la cantidad de un trabajo por año. 


\section{Tabla 1. Distribución de la producción realizada por año, país y categorías emergentes}

\begin{tabular}{|c|c|c|c|c|c|c|}
\hline \multirow{2}{*}{ Año } & \multicolumn{5}{|c|}{ Cantidad de trabajos por país } & \multirow[t]{2}{*}{ Temas } \\
\hline & Argentina & Brasil & Colombia & Francia & Perú & \\
\hline 2011 & & 11 & & & & $\begin{array}{l}\text { Alternancia y Desarrollo Local (3 textos); } \\
\text { Conformación de sujeto crítico (3 textos); } \\
\text { Instrumentos pedagógicos ( } 3 \text { textos); Análisis } \\
\text { histórico ( } 1 \text { texto); Inclasificable ( } 1 \text { texto). }\end{array}$ \\
\hline 2012 & 1 & 4 & 1 & 1 & & $\begin{array}{l}\text { Alternancia y Desarrollo Local (4 textos); } \\
\text { Instrumentos pedagógicos ( } 1 \text { texto); } \\
\text { Conformación de un sujeto crítico (1 texto); } \\
\text { Alternancia y educación y trabajo (1 texto). }\end{array}$ \\
\hline 2013 & & 7 & & & & $\begin{array}{l}\text { Instrumentos pedagógicos ( } 3 \text { textos); Análisis } \\
\text { histórico ( } 2 \text { textos); Alternancia y Desarrollo } \\
\text { Local ( } 1 \text { texto); Conformación de un sujeto } \\
\text { crítico (1 texto). }\end{array}$ \\
\hline 2014 & 1 & 14 & & & & $\begin{array}{l}\text { Conformación de un sujeto crítico (4 textos); } \\
\text { Alternancia y Desarrollo Local ( } 3 \text { textos); } \\
\text { Instrumentos pedagógicos ( } 3 \text { textos); } \\
\text { Alternancia y educación y trabajo ( } 2 \text { textos); } \\
\text { Análisis histórico ( } 2 \text { textos); Inclasificable ( } 1 \\
\text { texto). }\end{array}$ \\
\hline 2015 & 4 & 6 & & 1 & & $\begin{array}{l}\text { Conformación de un sujeto crítico ( } 3 \text { textos); } \\
\text { Alternancia y educación y trabajo ( } 3 \text { textos); } \\
\text { Análisis histórico ( } 2 \text { textos); Instrumentos } \\
\text { pedagógicos ( } 2 \text { textos); Alternancia y } \\
\text { Desarrollo Local ( } 1 \text { texto). }\end{array}$ \\
\hline 2016 & & 5 & & & 2 & $\begin{array}{l}\text { Alternancia y educación y trabajo ( } 2 \text { textos); } \\
\text { Instrumentos pedagógicos ( } 2 \text { textos); } \\
\text { Alternancia y Desarrollo Local ( } 2 \text { textos); } \\
\text { Análisis histórico ( } 1 \text { texto). }\end{array}$ \\
\hline 2017 & & 1 & & & & Análisis histórico (1 texto). \\
\hline TOTAL & 6 & 48 & 1 & 2 & 2 & \\
\hline
\end{tabular}

Fuente: Elaboración propia.

Francia ha sido el primer país en generar producción teórica respecto a la alternancia con los trabajos de Daniel Chartier, La spécificité des maisons familiales rurales d'édu- 
cation et d'orientation (1985), y André Duffaure, Educación, medio y alternancia (publicado originalmente en 1985 y traducido al español en 1994), lo cual es acorde con el hecho de que es el país en el cual surgió esta forma educativa. Llama la atención que, sin embargo, la producción sobre la alternancia no fue continua ni tampoco abundante en este país (a diferencia de Brasil). Tal como puede observarse en la Tabla 1, en el período 2011-2017 sólo encontramos dos textos producidos en el país en el cual surgió esta pedagogía.

Estos datos llaman la atención sobre todo al conectarlos con la cantidad de escuelas de alternancia existentes hoy en Francia (431, seguido por Brasil con 240 escuelas). A su vez, comparada con la fecha de surgimiento de la alternancia en dicho país (1935), podemos inferir que la reflexión teórica sobre esta forma educativa es muy posterior temporalmente a su diseño e implementación en la práctica, lo cual lleva a confirmar que el campo académico se interesó por esta pedagogía cuando ya se había expandido alrededor del mundo, sin haber generado conocimiento sobre las etapas iniciales de la misma. Incluso, los primeros trabajos sobre la pedagogía de la alternancia no provinieron del campo estrictamente académico, sino que fueron realizados por personas que pertenecían a este movimiento, tales como André Duffaure y Daniel Chartier. ${ }^{8}$

Finalmente, como vemos en la Tabla 1, Colombia y Perú son países en los cuales también hemos encontrado trabajos sobre la pedagogía de la alternancia, aunque la cantidad de producción es muy escueta (dos trabajos en Perú y uno en Colombia). Será interesante seguir la producción realizada en Perú, ya que allí se han producido recientemente dos textos, lo cual puede estar inaugurando una nueva área de conocimiento para ese país, que cuenta con escuelas de alternancia desde el año 2002.

En cuanto a la forma en que se fueron desarrollando las líneas temáticas a lo largo del tiempo, podemos ver que existe una constancia en cuanto a la presencia de todas las categorías temáticas desarrolladas en el apartado anterior. Las dos categorías que cuentan con mayor cantidad de textos (pedagogía de la alternancia y desarrollo local y desarrollo de los instrumentos pedagógicos) se encuentran presentes en todos los años de nuestro corte temporal (excepto en 2017).

8. Tanto Duffaure como Chartier fueron colaboradores de la Union Nationale des Maisons Familiales Rurales d'Education et d 'Orientation (UNMFREO), creada en 1942 para unir a todas la Maison de Francia, y se identifican como "los pioneros franceses" (Gimonet, 2009), pues han sido los primeros en describir pedagógica e institucionalmente los principales lineamientos de una forma escolar que comenzó desde la práctica y posteriormente fue teorizada. 
La categoría de la conformación de un sujeto crítico a través de la pedagogía de la alternancia (presente de forma continua en el tiempo desde 2011 a 2015) merece un comentario aparte, pues la misma aparece únicamente en trabajos brasileros. Creemos que esto tiene que ver con la experiencia de lucha y discusión iniciada por movimientos sociales, tales como el MST (Movimento dos Trabalhadores Sem Terra), el мав (Movimento dos Atingidos por Barragens), la СРТ (Comissão Pastoral da Terra) y los propios CEFFA (Centros Familiares de Formação por Alternância) que lograron tener representatividad en la sanción de la Lei de Diretrizes e Bases da Educação Nacional (LDB) de 1996, en la cual se utiliza ya el término Educação do Campo que apunta a un tipo de educación adecuada a la cultura y formas de producción del medio rural (da Silva, 2015). De esta manera, y en contraposición a lo que había ocurrido históricamente con la educación rural en Brasil (Almeida \& Germani, 2013), las escuelas de alternancia y las escuelas itinerantes de los movimientos sociales comienzan a identificarse como espacios de generación de aprendizajes ligados a la movilización política de los estudiantes.

En cuanto a las dos categorías que cuentan con menor cantidad de trabajos (análisis histórico y el vínculo entre educación y trabajo en la alternancia), en términos generales se puede observar que son líneas que han tenido continuidad a lo largo del tiempo, aunque cuentan con una menor cantidad de trabajos.

\section{Conclusiones}

El propósito de este artículo fue mapear la producción teórica del campo de la pedagogía de la alternancia tomando específicamente los trabajos realizados en el período 2011-2017. Este relevamiento nos permitió delinear las principales temáticas generadas por este campo hasta el momento, ver la forma en que estos temas se han desarrollado a lo largo del tiempo, visibilizar los países que concentran la mayor cantidad de producción (Brasil y, en menor medida, Argentina) y destacar algunas tendencias en cuanto a la distribución geográfica (por ejemplo, los inicios recientes de producción en este campo en Perú y la generación de una línea temática específica en Brasil, ligada a la alternancia y la conformación de un sujeto crítico y arraigado al medio rural).

Tal como mencionamos al inicio de este trabajo, el mapeo nos permite visualizar y ubicarnos espacialmente en el campo de la pedagogía de la alternancia, desplegan- 
do algunas articulaciones y debates aún implícitos. Así, la generación de categorías a través del agrupamiento semántico de los textos nos permitió recorrer las principales líneas temáticas de este campo. A su vez, podemos dar cuenta de que en cada categoría existen distintos enfoques; por ejemplo, en aquellos textos en que se toma un caso y se realiza una reconstrucción histórica, algunos trabajos subrayan las contradicciones que se dan entre las directrices generales de la alternancia y su aplicación en la práctica, mientras que otros se enfocan en los vínculos establecidos por determinados actores sociales. En la categoría de la conformación de un sujeto crítico y arraigado al medio rural predominan los trabajos que se centran en las luchas de los campesinos por permanecer en sus tierras, aunque también se destaca que el aspecto ligado a la co-gestión genera un tipo de subjetividad crítica (que recae no únicamente en los estudiantes sino también en las familias). Finalmente, en esta categoría encontramos trabajos que dan cuenta del potencial transformador de algunos instrumentos pedagógicos de la alternancia, como por ejemplo el plan de búsqueda.

También hemos podido localizar tensiones hacia el interior de algunas categorías, como es el caso de las reflexiones sobre los instrumentos pedagógicos de la alternancia, que son interpretados tanto como habilitadores de una resistencia cultural o bien como desactualizados respecto a algunas transformaciones acaecidas en el contexto rural más amplio. En la misma línea de destacar tensiones, la categoría de la pedagogía de la alternancia y la educación para el trabajo es tal vez la que muestra de manera más concisa un debate que atraviesa a casi todas las categorías, ya que la misma da cuenta de que la alternancia puede comprenderse como una pedagogía que forma trabajadores competentes para el mercado, pero que al mismo tiempo sirve para dar continuidad a determinados saberes de forma inter generacional. Si vinculamos este argumento con la categoría de la formación de sujetos críticos, vemos que la alternancia se conforma como un abanico que permite contemplar enfoques muy diversos respecto al vínculo entre la formación y las posibilidades de transformación de la realidad a través de lo educativo.

El mapeo nos ha permitido localizar una tensión que recorre el campo de la pedagogía de la alternancia relacionada con, por un lado, el potencial de esta pedagogía para la transformación de la realidad rural a través del arraigo, la permanencia en la tierra, la enseñanza de formas agroecológicas de producción, la participación de las familias, la aplicación de instrumentos pedagógicos que llevan a cuestionar y actuar sobre la realidad y, por otro, la alternancia como una pedagogía que permite extraer instrumentos para formar trabajadores competentes, técnicos o ingenieros agrope- 
cuarios que se inserten en el mercado de trabajo y que midan el desarrollo en función del crecimiento económico individual. Dicho de otro modo, el mapeo nos ha permitido observar que parecen convivir en este campo de investigación dos tendencias contrastantes: las referidas a "pedagogía de alternancia y emancipación” y las referidas a "pedagogía de alternancia y formación de empleados o trabajadores competentes".

Consideramos que este debate generado en la producción teórica sobre el campo de la alternancia representa una cuestión que también se da en la práctica y que nuestro equipo viene documentando a través del trabajo etnográfico mencionado al inicio de este artículo, en escuelas de la provincia de Buenos Aires. Resultaría interesante que nuevos trabajos puedan reconstruir cuáles y cuántos textos se enfocan más en uno u otro de estos dos "polos" del continuum "emancipación” / "sujeción”.

A su vez, se pueden hilar algunas articulaciones entre categorías, ya que, como vimos, muchas veces el foco puesto en la formación para el trabajo lleva a adentrarse en los instrumentos pedagógicos de la alternancia y viceversa, algunos trabajos se centran en los instrumentos pedagógicos para ver de qué forma éstos pueden ser usados en otros contextos, asociados a la formación profesional de técnicos o universitarios. Muchas veces, la entrada por los instrumentos pedagógicos desprende una reflexión sobre su potencial para el cuestionamiento de la propia realidad y sus posibilidades de transformación. También, reconstruir históricamente un caso de apertura de una escuela de alternancia puede llevar a analizar el desarrollo local, ya que hay varios actores que se movilizan para crear una escuela y esto, a su vez, se vincula con la conformación de sujetos críticos.

Finalmente, al observar las metodologías aplicadas en los trabajos podemos decir que encontramos una unicidad hacia el interior de las categorías relacionadas con la reconstrucción histórica y con los instrumentos pedagógicos. En ambas se trabaja con el estudio de caso para analizar en profundidad diversas dimensiones, tomando en cada categoría como caso ya sea la apertura de una escuela como algún instrumento pedagógico. Sin embargo, en cuanto a las demás categorías, podemos destacar algunos vacíos con relación a las metodologías utilizadas en los estudios sobre el campo de la alternancia, pues únicamente se implementan encuestas y entrevistas para recabar información, dejando de lado otros enfoques y técnicas. Esto podría incentivar a que futuros estudios contemplen usar otras metodologías para el análisis de diversos problemas o preguntas de investigación respecto a la alternancia, tal como el enfoque implementado en nuestro trabajo de campo, vinculado a la etnografía colaborativa y el análisis sociolingüístico de la interacción (Miano, 2017). 


\section{Lista de referencias}

Aldana, F. R. (2012). Análisis de la pedagogía de alternancia, como propuesta para generar desarrollo local en la zona rural estudio de caso con dos comunidades educativas del municipio de Machetá, Cundinamarca. Bogotá: Universidad JaverianaFacultad de Estudios Ambientales y Rurales de la Pontificia.

Almeida, J., \& Batista, C. R. (2011). As contribuições das Escolas Famílias Agrícolas do Semi-árido (EFAS) para (re)afirmação de identidades e manutenção dos jovens no campo. Trabajao presentado en el XV Seminário de Iniciação Científica de la Universidade do Vale do Itajaí, Brasil. Recuperado de http://www.xvsemic. esy.es/upload/2011/2011XV-031JAI493-100.pdf

Almeida, M., \& Germani, G. I. (2013). O território da educação do campo: As escolas escolas famílias agrícolas. Recuperado de http://observatoriogeograficoamericalatina.org.mx/egal14/Geografiasocioeconomica/Geografiaagricola/12.pdf

Altvater, E. (2012). El fin del capitalismo tal y como lo conocemos. España: El Viejo Topo. Amaral do, A. P. (2013). A pedagogia da alternância como práxis educativa na Escola Família Agrícola de Uirapuru: limites e potencialidades (Tesis de maestría). Universidade do Estado de Mato Grosso, Brasil. Recuperado de http://portal. unemat.br/media/oldfiles/educacao/docs/dissertacao/2013/ana_paula_do amaral.pdf

Araújo, S. R., Linhares, R., \& Teixeira, L. M. (2011). Pedagogía de la Alternancia y Agroecología: caminos para el desarrollo rural sustentable. Trabajo presentado en el V Congreso Iberoamericano sobre Desarrollo y Ambiente de la Red Iberoamericana de Economía Ecológica, Sociedad Internacional de Economía Ecológica, Asociación Argentina Uruguaya de Economía Ecológica y la Universidad Nacional del Litoral, Argentina. Recuperado de http://www.aimfr.org/ Archivos/Documentos/Ponencia\%2oBrasil.pdf

Assunção, C. A. G., \& Borges, R. (2012). Etnomatemática e Pedagogia da Alternância: Elo entre saber matemático e práticas sociais. Revista Latinoamericana de Etnomatemática, 5(1), 4-34. Recuperado de http://www.revista.etnomatematica.org/index.php/RevLatEm/article/view/35

Baraldo, N. (2010). Educación en y desde los movimientos sociales: ¿Nuevo objeto y nuevos abordajes en educación? Cuadernos de Educación, 8(8), 165-176. Recuperado de https://revistas.unc.edu.ar/index.php/Cuadernos/article/ view $/ 803 / 758$ 
Barbosa, J. A. (2014). Casa Familiar Rural. Expectativa para formação do empresário rural no município de Reserva/PR. Olhares y Trilhas, 19, 33-6o.

Batista, C. R. (2015). Estudo do lugar e Escolas Famílias Agrícolas: valorização do campo como conteúdo educativo e espaço de vivência cotidiana. Ateliê Geográfico, 9(1), 104-118. Recuperado de https://www.revistas.ufg.br/atelie/article/view/24249

Bencke, C., \& Costa, J. P. (2016). A proposta pedagógica do estágio de vivência na Escola Família Agrícola de Santa Cruz do Sul: uma alternativa de aprendizagem. Seminário Nacional de Pesquisa em Educação. Recuperado de http:// online.unisc.br/acadnet/anais/index.php/sepedu/article/view/14972

Bernartt, M. L., \& Pezarico, G. (2011). A pedagogia da alternância e seus referenciais teóricos metodológicos: construções a partir dos diálogos entre Brasil-África. Revista de Ciências Humanas, 12(19). Recuperado de http://revistas.fw.uri.br/ index.php/revistadech/article/view/346

Bock, M. L., \& Fagundes, E. M. (2014). Casa Familiar Rural: Contribuições para a educação dos jovens do campo no município de Pinhão/PR. Trabajo presentado en el IV Simpósio Nacional de Ensino de Ciência e Tecnologia de la Universidade Tecnológica Federal do Paraná Ponta Grossa, Brasil.

Boudjaoui, M., Clénet, J., \& Kaddouri, M. (2015). La formación en alternancia en Francia: entre prácticas sociales y objeto de investigación. Educar, 51(2), 239258. Recuperado de https://doi.org/10.5565/rev/educar.679

Calvo, C., \& Percíncula, A. (2012). Ligas Agrarias en Chaco y Corrientes. Experiencias de organización campesina en contextos de transformación territorial. De Prácticas y Discursos. Cuadernos de Ciencias Sociales, 1(1). Recuperado de http://ppct.caicyt.gov.ar/index.php/depracticasydiscursos/article/ view $/ 7138$

Cardoso, M. A., \& Hoff, S. (2013). A organização do trabalho didáctico na Escola Família Agrícola Rosalvo da Rocha Rodrigues: dados preliminares. Trabajo presentado en la XI Jornada do HISTEDbR, Universidade Estadual do Oeste do Paraná, Paraná, Argentina.

Carmo do, E., \& dos Prazeres, M. S. (2011). Casa Familiar Rural de Cametá: contribuições para o desenvolvimento das comunidades rurais no município de Cametá/PA. Trabajo presentado en el II Congresso Ibero-Americano de Política e Administração da Educação de la Associação Nacional de Política e Administração da Educação, Pontifícia Universidade Católica de São Paulo, Brasil. 
Recuperado de http://www.anpae.org.br/simposio2011/cdrom2011/PDFs/ trabalhosCompletos/comunicacoesRelatos/0177.pdf

Chartier, D. (1985). La spécificité des maisons familiales rurales d'éducation et dorientation. Revue Française de Pédagogie, 73, 23-30. Recuperado de https:// www.persee.fr/doc/rfp $0556-7807 \quad 1985$ num $73 \quad 1 \quad 1522$

Chaves, K., \& Foschiera, A. A. (2014). Práticas de educação do campo no Brasil: Escola Família Agrícola, Casa Familiar Rural e Escola Itinerante. Revista Pegada, 15(2), 76-94. Recuperado de http://revista.fct.unesp.br/index.php/pegada/ article/view $/ 3192$

Colatto, L. (2013). Pedagogia da alternância: Escola Família Agrícola. Castelo Branco Cientifica, 2(3), 1- 24 .

Conceição, J. J. X., \& da Costa, J. C. (2012). Caminhos da sustentabilidade: análise preliminar das práticas pedagógicas e o ensino aprendizagem dos alunos na escola família agrícola Rio Peixe-Balsas/MA. Geosaberes: Revista de Estudos Geoeducacionais, 3(5), 69-81.

Costa, M., \& Doula, S. M. (2014). Representações de Juventude na Escola Família Agrícola Paulo Freire. Acaiaca-MG. Revista Brasileira Multidisciplinar, 17(1), 59-76. Recuperado de https://doi.org/10.25061/2527-2675/ReBraM/2014. $\underline{\text { v17i1.6 }}$

Dinova, O. A. (1997). Escuelas de alternancia "Un proyecto de vida": educación rural por un campo mejor. Buenos Aires: GEEMA.

Duffaure, A. (1994). Educación, medio y alternancia. Buenos Aires: APEFA.

Feito, M. C. (2014). Ruralidades, agricultura familiar y desarrollo. Territorio del Periurbano Norte de la provincia de Buenos Aires. Buenos Aires: La Colmena.

Fernández, M. C., \& Welti, M. E. (2006). Historia y desarrollo de las escuelas de la familia agrícola en la provincia de Santa Fe: una singular articulación de saberes socialmente productivos. Revista de la Escuela de Ciencias de la Educación, $1,169-184$.

Frossard, A. C. (2014). Pedagogia da Alternância e Articulação dos Agentes Formativos de de Técnicos em Agropecuária: Interação entre Educação do Campo e Desenvolvimento Rural Sustentável em Nova Friburgo (Brasil) e Lobos (Argentina) (Tese doctoral inédita). Universidade Federal Rural do Rio de Janeiro, Rio de Janeiro.

Gimonet, J.-C. (2009). Lograr y comprender la pedagogía de la alternancia. Guatemala: Serviprensa. 
Glaser, B. G., \& Strauss, A. L. (1967). The Discovery of Grounded Theory: Strategies for Qualitative Research. Nueva York: Aldine.

Gluz, N. (2013). Las luchas populares por el derecho a la educación: experiencias educativas de movimientos sociales. Buenos Aires: Consejo Latinoamericano de Ciencias Sociales.

Gómez, M. V. (2011). El lugar de los contenidos relativos al espacio geográfico rural en las prácticas de enseñanza de una Escuela de la Familia Agrícola. Trabajo presentado en las VI Jornadas Nacionales sobre la Formación del Profesorado de la Universidad Mar del Plata, Argentina.

Gras, C. (2017). Expansión sojera y acaparamiento de tierras en Argentina. Desarrollo Económico. Revista de Ciencias Sociales, 57 (221), 149-163.

Heras, A. I., \& Miano, M. A. (2017). Educación, auto-organización y territorio. Revista Mexicana de Investigación Educativa, 22(73), 533-564.

Jahn, A. F., \& Presotto, S. (2013). Casa Familiar Rural: concepção de educação e realidade em Pérola D’Oeste. Trabajo presentado en la II Jornada Questão Agrária e Desenvolvimento de la Universidade do Paraná, Brasil. Recuperado de http://www.jornadaquestaoagraria.ufpr.br/trabalhos/uploads/artigojornadaquest_eoagr_aria.pdf

Kunrath, M. M. (2012). A proposta pedagógica da casa familiar rural de Porto Barreiro e o ensino na área do linguagens. Analecta, 13(1), 49-65. Recuperado de http://revistas.unicentro.br/index.php/analecta/article/view/3281

Lamas, H. (2016). La pedagogía de la alternancia. Apuntes Psicológicos, 1(2). Recuperado de https://revistas.upeu.edu.pe/index.php/ri_apsicologia/article/ view/869

Lima de, H. (2011). A pedagogia da alternância das Casas Familiares Rurais do Paraná. Trabajo presentado en el X Congresso Nacional de Educação de la Pontifícia Universidade Católica do Paraná, Brasil.

Lima, L., \& Freixo, A. (2012). Saberes e sabores do campo: relações entre conhecimentos científicos e tradicionais numa escola família agrícola do sertão da Bahia. Metáfora Educacional, 13, 21-35. Recuperado de http://www.valdeci.bio. $\mathrm{br} / \mathrm{pdf} / \mathrm{n} 13$ 2012/lima_freixo_saberes_e_n13_dez12.pdf

Lins, G., \& Cavalcante, L. (2011). Relação juventude rural-escola família agrícola. Discussões sobre o processo de escolarização e expectativas de futuro de jovens estudantes do ensino médio do sertão baiano. Trabajo presentado en el XVII Seminário de Iniciação Científica da Universidade Estadual de Feira de San- 
tana, Brasil. Recuperado de http://www2.uefs.br/semic/upload/2011/2011XV-o13GEO085-190.pdf

Lobo, E. M. (2011). Contributions of distance education the Pedagogy of Alternation. Trabajo presentado en el $17^{\circ}$ Congresso Internacional e Educaçao A Distancia de la Associação Brasileira de Educação a Distância, Brasil.

Lorenzo, C. (2012). La pedagogía de alternancia y el capital social. Estudio de caso del Серт $\mathrm{N}^{\circ} 5$ en Miranda, Rauch, provincia de Buenos Aires. Miríada: Investigación en Ciencias Sociales, 4(8), 125-144. Recuperado de http://p3.usal.edu.ar/ index.php/miriada/article/view/1417/

Melo de, J., Soares, M. J., \& Lucini, M. (2013). Formação por Alternância em Sergipe: O Estudo da Escola Família Agrícola de Ladeirinhas. Revista Tempos e Espaços em Educação, 6(10), 71-81. Recuperado de https://doi.org/10.20952/revtee. voio. 2295

Merenson, S., \& Garaño, S. (2015). Memorias rurales: avances y desafíos para los estudios sobre el pasado reciente en América Latina. Clepsidra, 2(3), 6-28.

Miano, A. (2017). La participación de las familias en la gestión escolar. Análisis de interacciones de un Consejo de Administración de una escuela de alternancia. Trabajo presentado en el $8^{\circ}$ Seminario Internacional de Investigación sobre Educación Rural del Consejo de Educación Inicial y Primaria, Uruguay.

Michi, N. (2010). Movimientos campesinos y educación. El Movimiento de los Trabajadores Rurales Sin Tierra y el Movimiento Campesino de Santiago del Estero-VC. Buenos Aires: El Colectivo.

Monge, Y. (2016). Formación en alternancia de jóvenes rurales. Buenos Aires: Noveduc. Mooney, P. R. (2002). El Siglo ETC. Erosión, Transformación Tecnológica y Concentración Corporativa en el Siglo 21. Montevideo: Nordan-Comunidad.

Nogar, A. G., \& Jacinto, G. P. (Eds.). (2010). Los espacios rurales. Aproximaciones teóricas y procesos de intervención en turismo rural. Buenos Aires: La Colmena.

Novais, J. (2012). A pedagogia da alternância e o debate da educação no/do campo no estado de Goiás. Revista Nera, 18, 7-20. Recuperado de http://revista.fct. unesp.br/index.php/nera/article/view/1334

Novais, M. P. (2014). A experiência da escola família agrícola de Quixabeira, no semiárido baiano / Experience schook family agricultural Quixabeira in Semiarid Baiano. Campo-Território: Revista de Geografia Agrária, 9(19), 531-543. Recuperado de http://www.seer.ufu.br/index.php/campoterritorio/article/ view/24551 
Oliva, D. E. (2016). Pedagogía de alternancia: una alternativa educativa para el nivel medio (C.E.P.T) del ámbito rural de la provincia de Buenos Aires, generadora de vínculos efectivos entre los jóvenes y el trabajo (Tesis de maestría). Facultad Latinoamericana de Ciencias Sociales, Buenos Aires. Recuperada de http://repositorio.flacsoandes.edu.ec/bitstream/10469/9325/2/TFLACSO-2016DEO.pdf

Oliveira de, A. (2013). Casa Familiar Rural e Pedagogia da Alternancia: uma metodologia viavel para campo. Pará: Universidade Federal do Pará.

Pacheco, J. C., \& Simonini, E. (2016). Narrando a construção de uma Escola Família Agrícola na trama de movimentos sociais. Educação em Perspectiva, 7(2), 283-302. Recuperado de https://educacaoemperspectiva.ufv.br/index.php/ ppgeufv/article/view/786

Padawer, A., Greco, J., \& Rodríguez, L. (2013). Educación y territorio en el so misionero: La escuela secundaria obligatoria en el contexto rural. Revista del Instituto de Investigaciones en Ciencias de la Educación, 33, 47-64. Recuperado de http://revistascientificas.filo.uba.ar/index.php/iice/article/view/1100

Pentecouteau, H. (2012). L'alternance dans une formation professionnelle universitaire. De l'idéal épistémologique aux contradictions pédagogiques. Reveu Internationale de Pédagogie de l'Enseignement Supérieur, 1(28). Recuperado de https://journals.openedition.org/ripes/605

Pereira, C (2015). Escola Família Agrícola no vale do Mucuri (MG): Uma experiência por meio de políticas públicas territoriais. Trabajo presentado en el XI Encontro Nacional da Associação Nacional de Pós-graduação em Geografia, Porto Alegre, Brasil. Recuperado de http://www.enanpege.ggf.br/2015/anais/arquivos/25/704.pdf

Pereira, T. (2016). Escola Família Agrícola de Sobradinho: O trabalho como princípio educativo para o desenvolvimento do campo do semiárido baiano. Ponencia presentada en el I Congreso Internacional da diversidade do Semiárido del Centro de Convenciones Raymundo Asfora, Campina Grande, Brasil.

Piatti, C. B. (2014). Pedagogia da alternância: espaços e tempos educativos na apropriação da cultura Pedagogía de la Alternancia: espacios y tempos educativos en la apropriación de la cultura. Boletim GEPEP, 3(5), 48-64. Recuperado de http://www2.fct.unesp.br/grupos/gepep/ze.pdf

Piovezana, L., \& Oliveira, M. (2012). Casa Familiar Rural: Uma educação diferenciada ao jovem em busca do desenvolvimento sustentável na agricultura familiar. Trabajo presentado en el IV Encontro em Educação Agrícola, Río de Janeiro. 
Queiroz Fraga, R., \& de Sousa, J. R. (2011). Pedagogia da alternância e prática educativa na educação do campo: experiência da escola família agrícola Dom Fragoso em Ceará, Brasil. Cisma: Revista de Humanidades y Ciencias Sociales, 6. Recuperado de http://periodicos.uesb.br/index.php/praxis/article/view/6669

Reis, J. P., \& Etges, V.E. (2016). Educação do Campo no Brasil: a experiência da Escola Família Agrícola de Santa Cruz do Sul-efasc. ReDES. Revista do Desenvolvimento Regional, 21(3), 300-319. Recuperado de https://online.unisc.br/seer/ index.php/redes/article/view/7528

Rotman, J. (2015). Escuela de la Familia Agrícola: lo que excede a la innovación educativa. Trabajo presentado en el VII Congreso Internacional de Investigación y Práctica Profesional en Psicología y XXII Jornadas de Investigación Décimo Encuentro de Investigadores en Psicología del mercosur de la Facultad de Psicología de la Universidad de Buenos Aires, Buenos Aires.

Sagastizábal, M. Á., Burin, D., Heras, A. I., \& Miano, M. A. (2006). El mapa conceptual como organizador epistémico y operativo de redes de investigación en Ciencias Sociales. Trabajo presentado en el II Congreso Internacional sobre mapas conceptuales de Institute for Human \& Machine Cognition, San José, Costa Rica.

Santana da Silva, L. (2012). Escola Família Agrícola do Sertão (EFASE): espaço de formação da classe trabalhadora e suas implicações na práxis educativa. Entrelaçando. Revista Electrónica de Culturas e Educação, 1(6), 75-93. Recuperado de http://www2.ufrb.edu.br/revistaentrelacando/component/phocadownload/ category $/ 185$ ? download $=193$

Santos, D. A., \& Cardel, L. M. (2011). Educação rural e as contradições do sistema de ensino: o caso da Escola Família Agrícola do Sertão. Educação em Revista, 12(2). Recuperado de http://www2.marilia.unesp.br/revistas/index.php/educacaoemrevista/article/view/2486

Santos, P. R., \& de Sousa, M. D. (2015). Os jovens egressos da Casa Familiar Rural de Açailândia: Fatores limitantes a actuação profissional de jovens camponeses. Brasil: Universidade Federal do Maranhão.

Schneider, S. (2013). Educação do campo e sustentabilidade: o caso da Escola Família Agrícola em Santa Cruz do Sul-RS. Atos de Pesquisa em Educação, 8(3), 964985. https://doi.org/10.7867/1809-0354.2013v8n3p964-985

Scopel, G., Passos, P., Palaoro, R., Dos Santos, C. C., \& Bernartt, M. L. (2011). Casas Familiares Rurais: histórico de implantação no Sudoeste do Paraná. Revista 
Synergismus Scyentifica UTFPR, 6(1). Recuperado de http://revistas.utfpr.edu. br/pb/index.php/SysScy/article/view/1263

Silva, E. M. (2013). Pedagogia da Alternância. Revista Terceiro Incluído, 3(2), 118-124. doi:10.5216/teri.v3i2.29802

Silva da, C. (2015). Escola família agrícola no Vale de Mucuri (MG): Uma experiencia por meio de políticas públicas territoriais. Trabajo presentado en el XI Encontro Nacional da Ampege.

Silva da, C., Andrade, K., \& Moreira, F. (2015). A retextualização no gênero Caderno da Realidade na Pedagogia da Alternância. Acta Scientiarum. Language and Culture, 37(4), 359-369. https://doi.org/10.4025/actascilangcult.v37i4.25050

Sobreira, M. F., \& da Silva, L. H. (2014). Vida e construção do conhecimento na Pedagogia da Alternancia. Revista Eletrônica de Educação, 8(2), 212-227. Recuperado de http://www.reveduc.ufscar.br/index.php/reveduc/article/view/915

Texeira, D. (2013). O papel da Escola Família Agrícola de Santa Cruz do Sul na permanência do jovem no campo. Porto Alegre: Universidade Federal do Rio Grande do Sul.

Teixeira, E. S., \& Antunes, L. C. (2011). Casas Familiares Rurais e desempenho escolar: um estudo na região sudoeste do Paraná. Revista Diálogo Educativo, 11(34), 951-969.

Teixeira, E. S., Bernartt, M. L., \& Trindade, G. A. (2008). Estudos sobre Pedagogia da Alternância no Brasil: revisão de literatura e perspectivas para a pesquisa. Educação e Pesquisa, 34(2), 227-242. Recuperado de https://doi.org/10.159o/ $\underline{S_{1517}-97022008000200002}$

Teubal, M. (2006). Expansión de la soja transgénica en la Argentina. Global Development And Environment Institute. Recuperado de https://ase.tufts.edu/gdae/ Pubs/rp/wg/AgricultureBook_Span/PromesasPeligrosCh4Teubal.pdf

Valadão, J., Neto, J. R., \& de Andrade, J. (2017). Bases sociotécnicas de uma tecnologia social: o transladar da pedagogia da alternância em Rondônia. Organizações \& Sociedade, 24(80), 89-114. Recuperado de https://doi.org/10.1590/19842230805

Zanuncio, S., \& Zanuncio, F. (2015). Escola Família Agrícola, Pedagogia da Alternância, Qualidade de Vida e Perspectivas Futuras dos Alunos: O Caso da EFA/Castelo, Espírito Santo. Revista Cesumar Ciências Humanas e Sociais Aplicadas, 2o(1). 